\title{
Laparoscopic Resection of a Jejunal Inflammatory Fibroid Polyp that Caused Occult Gastrointestinal Bleeding, Diagnosed via Capsule Endoscopy and Double-Balloon Enteroscopy: A Case Report
}

\author{
Chizu Kameda', Hideaki Miwa², Ryohei Kawabata', Daiki Marukawa', Masahiro Murakami', Shingo Noura', Junzo Shimizu'1 and \\ Junichi Hasegawa' \\ Departments of ${ }^{1}$ Surgery, ${ }^{2}$ Pathology, Osaka Rosai Hospital, Osaka, Japan
}

An inflammatory fibroid polyp (IFP) is a mesenchymal tumor of the gastrointestinal tract. IFPs in the small intestine are the most frequently detected with symptoms, such as abdominal pain and tarry stool due to intussusception. Accordingly, few studies have reported jejunal IFP as a cause of occult gastrointestinal bleeding (OGIB) diagnosed via both of capsule endoscopy (CE) and doubleballoon enteroscopy (DBE). A 68-year-old woman presented with a progression of anemia and a positive fecal occult blood test result. Esophagogastroduodenoscopy and total colonoscopy findings were unremarkable. CE revealed a tumor with bleeding in the jejunum. DBE also revealed a jejunal polypoid tumor. Bleeding from the tumor seemed to have caused anemia. The patient underwent partial laparoscopic resection of the jejunum, including resection of the tumor. The tumor was histopathologically diagnosed as IFP. To our knowledge, this is the first reported case of laparoscopic resection of jejunal IFP with OGIB diagnosed via CE and DBE preoperatively. Clin Endosc 2018;51:384-387

Key Words: Inflammatory fibroid polyp; Capsule endoscopy; Double balloon enteroscopy; Laparoscopic surgery

\section{INTRODUCTION}

Occult gastrointestinal bleeding (OGIB) can occur anywhere throughout the gastrointestinal tract and is defined as gastrointestinal persistent or recurrent bleeding with no visible symptoms to the patient or physician and no definitive source, and usually identified via initial esophagogastroduodenoscopy or colonoscopy. Thus far, several reports have shown the usefulness of capsule endoscopy (CE) for evaluating patients with OGIB. ${ }^{2,3}$ Indeed, at present, OGIB is the foremost indication

Received: October 13, 2017 Revised: January 4, 2018

Accepted: January 15, 2018

Correspondence: Chizu Kameda

Department of Surgery, Osaka Rosai Hospital, 1179-3 Nagasonecho, Kita, Sakai, Osaka 591-8025, Japan

Tel: +81-72-252-3561, Fax: +81-72-255-3349, E-mail: chizuk923@gmail.com

ORCID: https://orcid.org/0000-0001-9778-4224

(c) This is an Open Access article distributed under the terms of the Creative Commons Attribution Non-Commercial License (http://creativecommons.org/ licenses/by-nc/3.0) which permits unrestricted non-commercial use, distribution and reproduction in any medium, provided the original work is properly cited. for $\mathrm{CE}$, with a high detection rate and low retention rate. ${ }^{4}$

Inflammatory fibroid polyps (IFPs) are benign and neoplastic lesions that can occur throughout the gastrointestinal tract. IFP is often found in the stomach and is rarely occurs in the jejunum. ${ }^{5}$ IFP in the small intestine is most frequently detected with initial symptoms such as abdominal pain and tarry stool because of intussusception. Thus, emergency surgery is often performed. ${ }^{6}$ However, little has been reported on the cases of laparoscopic surgery for IFPs as the focus of OGIB in the small intestine that are diagnosed using CE and double-balloon enteroscopy (DBE). We had a case of jejunal IFP with OGIB detected on CE and DBE that was successfully treated with laparoscopic resection.

\section{CASE REPORTS}

A 68-year-old woman presented with a 2-month history of progression of anemia and positive fecal occult blood test 

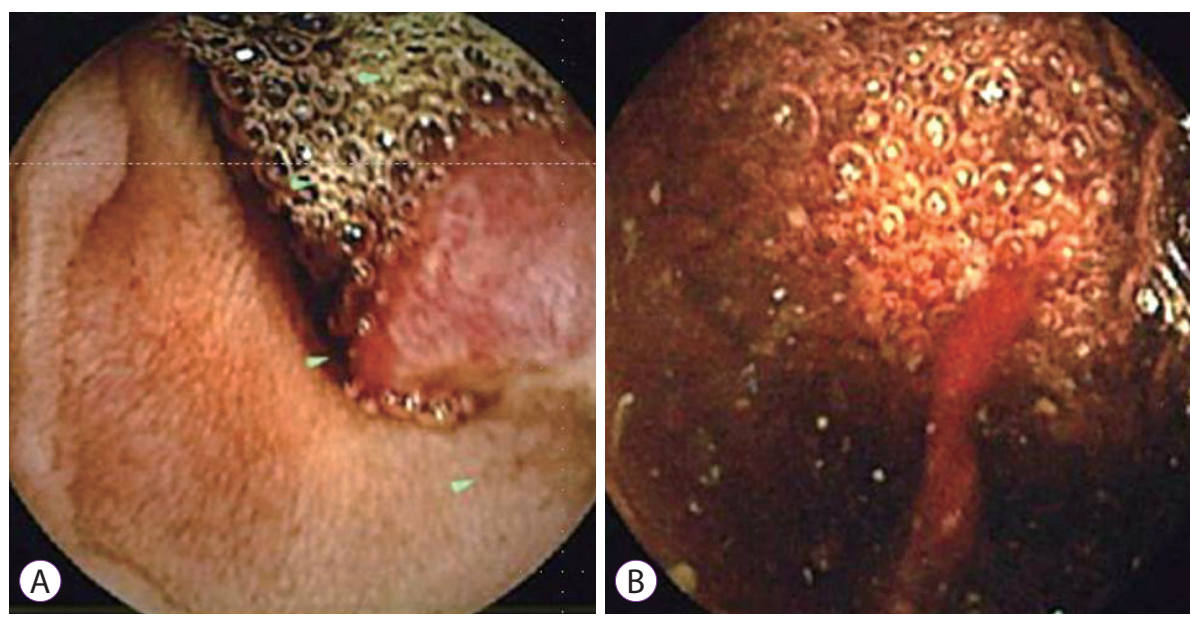

Fig. 1. (A, B) Video capsule endoscopic image showing the tumor with bleeding in the distal jejunum.

findings. She had underlying medical conditions, including diabetes mellitus and chronic renal failure on dialysis, and a history of right nephrectomy for renal cancer, breast-conserving surgery for right breast cancer, appendectomy for appendicitis, and right oophorectomy for ovarian cyst. She had no complaints of abdominal pain, melena, or weight loss. A physical examination showed that she had anemic conjunctivae. In addition, she was obese, with a body mass index of $29 \mathrm{~kg} / \mathrm{cm}^{2}$, and she had a scar caused by several operations in the lower-middle part of the abdomen, right lower quadrant of the abdomen, and right lateral abdomen. The scars in the lower middle part of the abdomen and right lateral abdomen had an incisional hernia. Laboratory examination showed that her serum hemoglobin level had decreased from 9.2 to 6.6 $\mathrm{mg} / \mathrm{dL}$ over 2 months. These findings suggested gastrointestinal bleeding. Thus, esophagogastroduodenoscopy and total colonoscopy were performed, neither of which was useful for identifying any focus of active bleeding. Subsequently, CE was performed to determine the cause of OGIB, and a mass with bleeding in the jejunum was identified (Fig. 1A, B). DBE confirmed the mass to be approximately $20 \mathrm{~mm}$ in diameter with erosion in the jejunum, and no signs of obstruction (Fig. 2). Although the diagnosis on the basis of an endoscopic biopsy was jejunitis and no neoplastic changes were observed, the anemia seemed to have been caused by bleeding from the tumor. Therefore, surgery was recommended for the diagnosis and treatment of the tumor.

Laparoscopic resection was selected. A $12-\mathrm{mm}$ port for the laparoscope was inserted into the umbilical site, and two 5-mm ports were inserted in the left lateral abdomen (Fig. 3A). Laparoscopy confirmed the preoperative tattooing localization of a tumor $150 \mathrm{~cm}$ from the ligament of Treitz. No specific findings were found in the serosa (Fig. 3B). The tattooed part of the jejunum was removed extracorporeally, and partial resection of the jejunum and functional end-to-end anastomo-

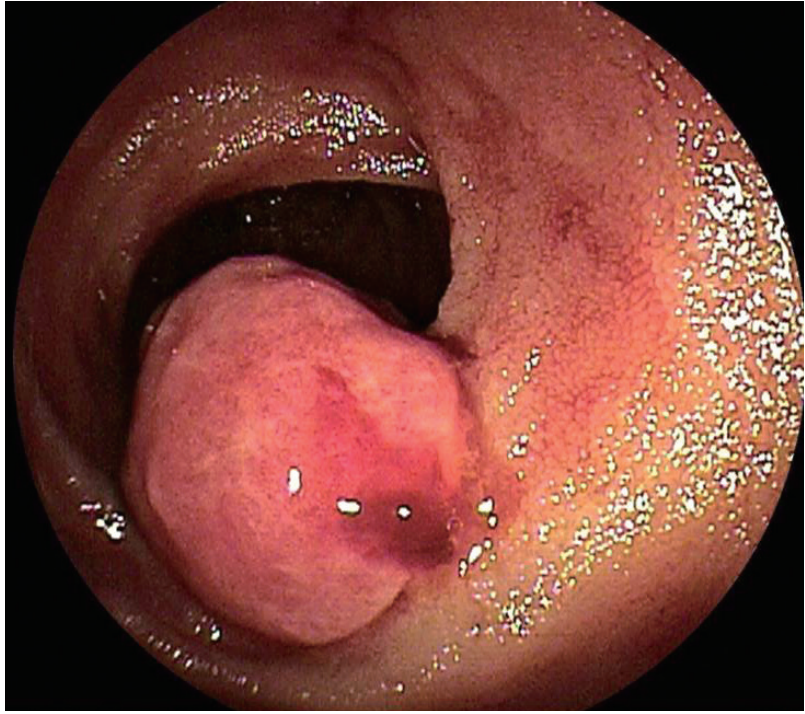

Fig. 2. Double balloon enteroscopy showing a polypoid mass in the distal jejunum.

sis were performed. The macroscopic findings of the resected specimen showed a partially dark-red polypoid mass in the jejunal wall, measuring $12 \times 10 \times 10 \mathrm{~mm}$ in size (Fig. $4 \mathrm{~A}$ ). Histological examination revealed the tumor to be located in the submucosa mainly and an apparently-destroyed mucosal layer. The tumor was not a neoplastic lesion but a granulomatous one with erosion, edema, proliferation of the small vessels and infiltration of rich eosinophils and lymphocytes (Fig. 4B, C). These findings coincided with IFP developing in the jejunum. After surgery, the patient's recovery was uneventful, and she has remained well since. Her anemia ceased, and her blood hemoglobin level has remained in the normal range in the 18 months following her discharge. 


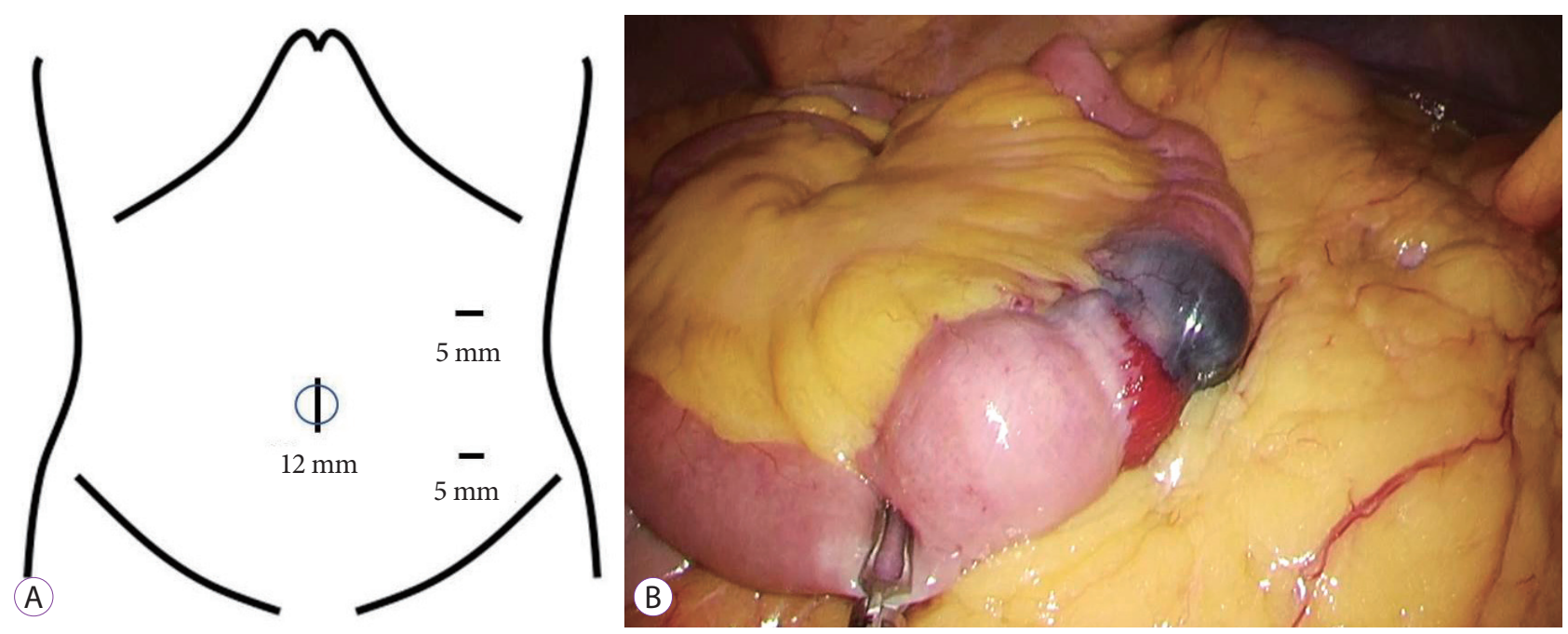

Fig. 3. (A) A 12-mm initial access site established at the umbilicus by using the Hasson open technique. A 12-mm trocar and two 5-mm trocars are inserted as indicated. (B) Laparoscopic image confirming the preoperative tattooing of the tumor in the jejunum.
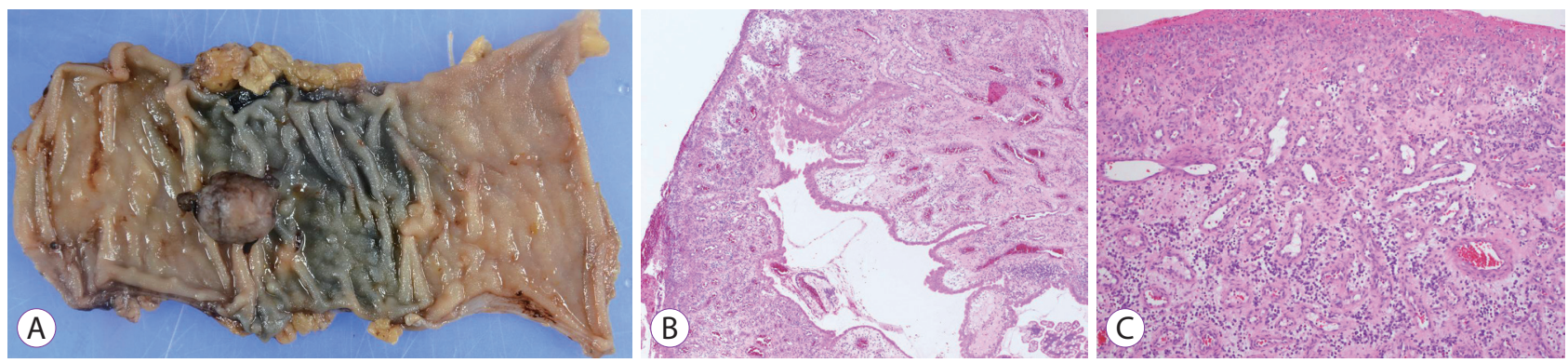

Fig. 4. Macroscopic and histopathological examinations of the surgical specimen. (A) The macroscopic findings show a partially dark-red polypoid mass in the jejunal wall. The histopathological examination result shows that the tumor consisted of fibrous granulation tissue (hematoxylin and eosin [H\&E] staining, original magnification $\times 40)(B)$ with mild infiltration of eosinophils and lymphocytes. $(H \& E$, original magnification $\times 100)(C)$.

\section{DISCUSSION}

IFP was first described by Vanek as gastric submucosal granuloma with eosinophilic infiltration. ${ }^{7}$ The symptoms caused by IFP are various. The patients often complain of abdominal pain, weight loss, melena, dyspeptic symptoms and obstruction depending on the location and size of the IFP lesion. ${ }^{8}$ Most IFPs in the small intestine reported in the literature to date have been found to cause bowel obstruction as a result of intussusception. ${ }^{9}$ However, only a few cases of IFP in the small intestine with OGIB on endoscopic and imaging tests have been reported. ${ }^{10,11}$ Sami presented an overview of the literature on cases of intussusception due to IFP that were reported between 1976 and 2011. He found that IFPs measured between 1.5 and $11 \mathrm{~cm}$ (median, $3.5 \mathrm{~cm}$ ) in diameter at the time of diagnosis. ${ }^{12}$ Our case, which had a diameter of $1.2 \mathrm{~cm}$, was macroscopically smaller than any IFP in that review. Therefore, the IFP in our case might have been identifiable on CE even without subjective symptoms.
Conventional endoscopic examinations for the small intestine are limited because of difficulty in observing the jejunum and ileum. Therefore, $\mathrm{CE}$ is a useful noninvasive diagnostic tool and has proved to be an extraordinarily safe device with few adverse events and complications. ${ }^{2}$ The American society for Gastrointestinal Endoscopy guideline recommends $\mathrm{CE}$ as the first-choice diagnostic tool to evaluate the small bowel for OGIB if no contraindications are present. ${ }^{13} \mathrm{CE}$ allows the exploration of the entire small intestine in nearly $85 \%$ of the cases with OGIB in a meta-analysis. ${ }^{2,14}$ Accordingly, several studies have reported that the diagnostic yield of $\mathrm{CE}$ in patients with OGIB is $46 \%-81 \%{ }^{15,16}$ However, Han et al. reported that the miss rate of $\mathrm{CE}$ for the small intestine was $16.5 \%{ }^{17}$ Our present patient with progression of anemia was found to have a tumor in the jejunum on $\mathrm{CE}$, although the detection of small bowel tumors with no subjective symptoms is rare owing to the difficulty of such a diagnosis. Shimada et al. evaluated the factors associated with positive CE findings in patients with OGIB, showing that a low hemoglobin level was associated with an 
increased prevalence of positive CE findings. ${ }^{4}$

We performed not only CE but also DBE to obtain detailed information on the tumor in the jejunum. DBE is another novel endoscopic technique that allows for deep insertion of an endoscope for evaluating the small bowel mucosa. ${ }^{18}$ In addition, DBE enables tumor biopsy to obtain a final diagnosis prior to surgery and marking of the surgical site to identify the lesion during surgery and perform therapeutic procedures for small bowel lesions. In our case, DBE enabled the identification of a site for a tumor biopsy and preoperative localization of the tumor by tattooing. Imaoka et al. evaluated the characteristics of small bowel tumors detected using DBE and found that this procedure was a historical diagnosis of all cases investigated. ${ }^{19}$ In the present case, DBE could not confirm the accurate pathological diagnosis of jejunal tumor prior to surgery because the IFP was a submucosal tumor. However, we could still prepare for the operation in accordance with the diagnosis made from the tumor biopsy and successfully performed laparoscopic resection of the tumor, which has low invasiveness, owing to the case of identification of the tumor location during the operation.

Many studies have demonstrated the minimal invasiveness of laparoscopic surgery as compared with open surgery, with such results as reduced postoperative pain, less blood loss, shorter length of hospital stay, fewer postoperative complications, and better cosmetic outcome. Consequently, the use of laparoscopic techniques has rapidly increased worldwide. ${ }^{20}$ In our case, the patient was obese and had incisional hernia due to several open surgeries, in addition to underlying diabetes mellitus and chronic renal failure on dialysis. Therefore, owing to laparoscopic resection, the risk of complications such as surgical site infection and incisional hernia in the present case was reduced.

We searched the English and Japanese-language medical literatures related to IFP with OGIB in the small intestine diagnosed with endoscopy that were published between 1997 and August 2017, using PubMed and Japan the Medical Abstracts Society databases. The key words used were "inflammatory fibroid polyp in small intestine". We found a total of six reports on IFPs in the small intestine detected using endoscopy. As far as we know, there was no report wherein the IFP diagnosed by both CE and DBE before laparoscopic resection.

In conclusion, we had a case of jejunal IFP that caused OGIB diagnosed using CE and DBE. CE and DBE are useful procedures for visualizing the entire small intestine in case of OGIB and can aid in the performance of laparoscopic surgery, which is the preferred treatment for patients.
Conflicts of Interest

The authors have no financial conflicts of interest.

\section{REFERENCES}

1. De Leusse A, Landi B, Edery J, et al. Video capsule endoscopy for investigation of obscure gastrointestinal bleeding: feasibility, results, and interobserver agreement. Endoscopy 2005;37:617-621.

2. Liao Z, Gao R, Xu C, Li ZS. Indications and detection, completion, and retention rates of small-bowel capsule endoscopy: a systematic review. Gastrointest Endosc 2010;71:280-286.

3. Shim KN, Moon JS, Chang DK, et al. Guideline for capsule endoscopy: obscure gastrointestinal bleeding. Clin Endosc 2013;46:45-53.

4. Shimada S, Watanabe T, Nadatani Y, et al. Clinical factors associated with positive capsule endoscopy findings in patients with obscure gastrointestinal bleeding: a single-center study. Scand J Gastroenterol 2017;52:1219-1223.

5. Abboud B. Vanek's tumor of the small bowel in adults. World J Gastroenterol 2015;21:4802-4808.

6. Kang SH, Kim SW, Moon HS, et al. Inflammatory fibroid polyp in the jejunum causing small bowel intussusception. Ann Coloproctol 2015;31:106-109.

7. Vanek J. Gastric submucosal granuloma with eosinophilic infiltration. Am J Pathol 1949;25:397-411.

8. Wysocki AP, Taylor G, Windsor JA. Inflammatory fibroid polyps of the duodenum: a review of the literature. Dig Surg 2007;24:162-168.

9. Smith-Chakmakova F, Liu YJ, Karamchandani DM. Inflammatory fibroid polyp of the ileocecal valve: case report, review, and recent advances. Ann Clin Lab Sci 2015;45:441-445.

10. Kim SG, Choi KD, Kim JS, Jung HC, Song IS. Ileal inflammatory fibroid polyp: a rare cause of obscure gastrointestinal bleeding diagnosed by wireless capsule endoscopy. Dig Dis Sci 2006;51:906-908.

11. Takeshita K, Kashiwagi K, Ishibashi Y, Kanai T. An ileal inflammatory fibroid polyp treated by endoscopic resection. Intern Med 2016;55:29012902.

12. Akbulut $\mathrm{S}$. Intussusception due to inflammatory fibroid polyp: a case report and comprehensive literature review. World J Gastroenterol 2012;18:5745-5752.

13. ASGE Standards of Practice Committee, Fisher L, Lee Krinsky M, et al. The role of endoscopy in the management of obscure GI bleeding. Gastrointest Endosc 2010;72:471-479.

14. Lepileur L, Dray X, Antonietti M, et al. Factors associated with diagnosis of obscure gastrointestinal bleeding by video capsule enteroscopy. Clin Gastroenterol Hepatol 2012;10:1376-1380.

15. Lewis BS, Eisen GM, Friedman S. A pooled analysis to evaluate results of capsule endoscopy trials. Endoscopy 2005;37:960-965.

16. Das K, Sarkar R, Dasgupta J, et al. Obscure GI bleeding in the tropics: impact of introduction of double-balloon and capsule endoscopies on outcome. Gastrointest Endosc 2010;72:292-300.

17. Han JW, Hong SN, Jang HJ, et al. Clinical efficacy of various diagnostic tests for small bowel tumors and clinical features of tumors missed by capsule endoscopy. Gastroenterol Res Pract 2015;2015:623208.

18. Kim TJ, Kim ER, Chang DK, Kim YH, Hong SN. Comparison of the efficacy and safety of single- versus double-balloon enteroscopy performed by endoscopist experts in single-balloon enteroscopy: a single-center experience and meta-analysis. Gut Liver 2017;11:520-527.

19. Imaoka H, Higaki N, Kumagi T, et al. Characteristics of small bowel tumors detected by double balloon endoscopy. Dig Dis Sci 2011;56:23662371.

20. Viñuela EF, Gonen M, Brennan MF, Coit DG, Strong VE. Laparoscopic versus open distal gastrectomy for gastric cancer: a meta-analysis of randomized controlled trials and high-quality nonrandomized studies. Ann Surg 2012;255:446-456. 